\title{
Challenges and successes in engaging citizen scientists to observe snow cover: from public engagement to an educational collaboration
}

\author{
Susan E. Dickerson-Lange, Karla Bradley Eitel, Leslie Dorsey, \\ Timothy E. Link and Jessica D. Lundquist
}

Abstract

Keywords

Context
Whereas the evolution of snow cover across forested mountain watersheds is difficult to predict or model accurately, the presence or absence of snow cover is easily observable and these observations contribute to improved snow models. We engaged citizen scientists to collect observations of the timing of distributed snow disappearance over three snow seasons across the Pacific Northwest, U.S.A. . The primary goal of the project was to build a more spatially robust dataset documenting the influence of forest cover on the timing of snow disappearance, and public outreach was a secondary goal. Each year's effort utilized a different strategy, building on the lessons of the previous year. We began by soliciting our professional networks to contribute observations via electronic or paper forms, moved to a public outreach effort to collect geotagged photographs, and finally settled on close collaboration with an outdoor science school that was well-positioned to collect the needed data. Whereas the outreach efforts garnered abundant enthusiasm and publicity, the resulting datasets were sparse. In contrast, direct collaboration with an outdoor science school that was already sending students to make weekly snow observations proved fruitful in both data collection and educational outreach. From a data collection standpoint, the shift to an educational collaboration was successful because it essentially traded wide spatial coverage combined with sparse temporal coverage for dense temporal coverage at a single, but important location. From a public engagement standpoint, the partnership allowed for more intensive participation by more people and enhanced the science curriculum at the collaborating school.

Citizen science; Science education

The spatial pattern of snow cover across a forested mountain watershed is easily observed by outdoor enthusiasts who readily make note of snow disappearance patterns at a range of scales when they avoid skiing into tree well, change a hiking route after encountering a snow field, or capture a photograph of patchy snow across a valley. Whereas these patterns are obvious to an observer, reproducing such a pattern via a computer model is an ongoing research challenge [Essery et al., 
2009]. Hydrologic models attempt to incorporate the physical influences of forest cover on snow processes, but are limited by approximations of physical processes that are based on site-specific field data that may lack transferability to different climates and forests [Clark et al., 2015]. In particular, observational data documenting snow cover patterns, which represent the spatial variability of the timing of snow disappearance in forested mountain watersheds are needed because the net effect of forest cover on snow can be to delay or accelerate the date of snow disappearance relative to open areas [Lundquist et al., 2013]. Subsequently, the timing of snow disappearance influences soil moisture availability, streamflow amount, and stream temperature during the dry summer season in the mountains of the American West [Ford et al., 2013; Lundquist, Dettinger and Cayan, 2005]. By capitalizing on the presence of hikers, skiers, snowmobilers and others who are often in "the right place at the right time" to observe snow cover patterns, a citizen science approach has the potential to yield critical data to further understanding of snow processes and to test watershed models.

Improved estimates of the effect of forest management actions, such as thinning or clear-cutting, on the timing of snow disappearance are relevant to land managers who consider multiple objectives when making decisions, including forest resilience to insect outbreaks, aquatic ecosystem health, fire fuels management, and adaptation to climate change. Previous work has supported the creation of forest gaps as a way to retain snow on the landscape [Dickerson-Lange et al., 2015] and thus shift the timing of peak spring streamflows to later in the year (i.e., due to later snowmelt [Ellis, Pomeroy and Link, 2013]) to the benefit of late season water supply and ecosystem health. However, this management strategy is likely to have the intended effect only within a specific set of conditions. The magnitude and direction of forest influences on snow processes, such as snow accumulation and melt, depends on winter climate [Lundquist et al., 2013], topographic position such as north- versus south-facing slopes [Ellis, Pomeroy and Link, 2013], and forest composition and density [Kittredge, 1953].

Thus, in order to support an ongoing effort to develop management-relevant maps of where forest is likely to delay versus accelerate snow disappearance in the Pacific Northwest, U.S.A., additional field data documenting the differential timing of snow disappearance between forested and non-forested areas are needed. Unfortunately, such observational data are limited in both temporal and spatial coverage because field campaigns are expensive, time-consuming and hindered by access issues [Elder and Cline, 2003]. Furthermore, satellite methods to detect snow cover are uncertain within forests because the tree canopies obscure the ground surface conditions [Raleigh et al., 2013]. Therefore, even after incorporating field data from a network of collaborating institutions across the region into this project there are still critical data gaps in parts of the region including eastern Washington, eastern Oregon, and central Idaho, each of which has unique climate and forest conditions (Figure 1a).

Since the beautiful mountain landscapes that attract outdoor enthusiasts are home to the same locations in which additional forest-snow observations are needed, engaging the people who already recreate there in collecting data seems ideal. Furthermore, with a particular interest in the snowmelt season (i.e., spring and summer), skiers who are seeking spring snow and hikers who are seeking snow-free routes are well-positioned to collect data at the optimal time to capture differential timing of snow disappearance across forested and open areas. 
This model of opportunistic citizen science has previously proven successful in locations that people are motivated to visit, such as to see the wildflowers bloom on Mount Rainier each summer [Wilson et al., 2015]. Many successful citizen science projects leverage large numbers of observations to develop robust spatial or temporal resolution, and to reduce the uncertainty associated with amateur data collectors. For example, one million bird observations are submitted from around the world each month as part of the bird observation programs implemented by the Cornell Lab of Ornithology [Bonney et al., 2009]. The utilization of citizens to collect large numbers of data points is typically associated with minimal training, and this strategy has been described as utilizing citizens as sensors [Haklay, 2013] or as crowdsourcing in a framework of the possible levels of engagement of citizen scientists (hereafter "level 1").

In the same model [Haklay, 2013], the next level of participation (hereafter, "level 2") involves more training in order to develop interpretation skills, relying on participants to complete some basic interpretation while collecting data. The data collected by citizens who are participating in a level 2 project are likely to be higher quality, but since data collection involves more training and therefore is more time and energy-intensive, a level 2 project is also likely to engage fewer people. Many successful school-based citizen science programs occur at this level, in which teachers provide instruction, a framework for participation, and assistance in making decisions about when and where to collect data [Rock and Lauten, 1996; Eick et al., 2008]. Such programs have been shown to generate high quality data [Peckenham and Peckenham, 2014; Lawless and Rock, 1998; Galloway, Tudor and Haegen, 2006] in addition to improving educational outcomes [Bingaman and Eitel, 2010; Schon et al., 2014].

Although citizen science arguably has its roots in ecology in general [Silvertown, 2009], and surveys in particular, many previous efforts have successfully incorporated citizen science in hydrology [Buytaert et al., 2014] These investigations have focused primarily on water quality [Peckenham and Peckenham, 2014] or water quantity in the form of precipitation [CoCoRaHS, 2015] or streamflow [Lowry and Fienen, 2013]. Previous snow hydrology investigations to formally utilize citizen scientists are limited, as far as we are aware, to a snow study that involves tweeting point snow depth values (http:/ / scistarter.com/project/205SnowTweets?tab=project, accessed 14 May 2015) but citizen observations of snow presence and conditions are certainly included in backcountry snow reports utilized by the recreation community (e.g., http:/ / www.wta.org/go-hiking/trip-reports, accessed 14 May 2015) and on social media.

In addition to providing a method to collect spatially distributed data, citizen science has been shown to have key benefits for public engagement. Projects have yielded positive social benefits by enhancing community collaboration [Borden et al., 2007] increasing participation in locally-relevant environmental issues [Cooper et al., 2007] and guiding development of land management strategies [Rosenberg et al., 2003]. Bonney et al. [2014] further predict that increased implementation of citizen science holds potential for strengthening the relationship between scientific efforts and society. Since the ongoing forest-snow investigation described here has potential management applications, communication with land managers and public stakeholders is a key project goal, and a citizen science approach provides an avenue to engage the public in addition to collecting data. 



Figure 1. Map of the Pacific Northwest showing collaborating field sites (gray dots) and McCall Outdoor Science School (MOSS, black triangle) (a). Photograph of MOSS students estimating snow cover in different directions (b). Example graph of average snow cover in open and forested areas at Quad Forest, one of the MOSS field sites. Graphs for each site were updated in real-time on a website that was linked to the online form for data collection (c).

Thus, in March 2012, we moved forward with enthusiasm for the idea but few analogous models for utilizing citizen science to observe snow cover and develop a more spatially robust dataset for the Pacific Northwest.

Objective \& methods
Motivated by the clear congruence between our data needs, public outreach goals, and the already-occurring citizen forays into the mountains, we began a citizen science project three years ago to document the timing of snow disappearance across the mountains of the Pacific Northwest. The project was initiated by a team of two university researchers at the University of Washington, and evolved into collaboration between three universities and a K12 outdoor science school. We detail our citizen science effort from the perspective of both researchers and educators, using the writing voice of the research institutions but including co-authors from both sides of the educational collaboration.

The project evolved substantially each year as we incorporated lessons from the successes and failures of the previous year. This citizen science effort was motivated first and foremost by a critical need for more distributed data across the greater Pacific Northwest region, rather than as a study of citizen science itself. Thus, 
evaluation efforts focused on quantifying the usable data received each year in an effort to understand what was working for meeting our scientific goals. To consider the success of the project in terms of public outreach, we informally assessed the experience of participants through written comments included in data submission and follow-up email communications and made adjustments accordingly.

Whereas the first two years were effective in increasing engagement with public stakeholders, the participation model that we utilized did not ultimately match our primary data needs. We therefore re-assessed the requirements for usable data and shifted our focus from public engagement to collaboration with an outdoor science school on the third year, effectively trading spatial coverage for usable temporal resolution. This tradeoff between breadth and depth has been previous identified as a potential difficulty facing researchers who delve into citizen science [Riesch, Potter and Davies, 2013].

However, we found that the decision to change our focus from widespread participation to a single site was a turning point in the overall success of the project and a model from which the project could again expand in spatial scope. We therefore present the relevant experiences and lessons from three years of citizen science with a particular emphasis on the evolution of the project from public outreach to educational collaboration.

We originally envisioned the citizen science project as a crowdsourcing effort [Haklay, 2013; Howe, 2006] in which numerous participants, who were in the mountains during the spring and summer for other purposes (e.g. recreation), would document observations of the differential timing in snow disappearance between forested and open areas. In essence, we needed comparisons of snow presence between forested areas and meadows or clearings that would allow us to determine 1) where snow lasted longer in the forest versus an equivalent open area (or vice versa), and 2) how long the snow persisted in the forest after disappearing from the clearing (or vice versa).

In year one, we contacted our personal networks to solicit "beta testing" from people we knew who were likely to be in the mountains during the melt season, including researchers, a national park scientist, and mountaineers. We developed a questionnaire to record time and location details, relative snow presence, and opportunities for additional observations and feedback. We provided basic instructions in email communications and two formats for responding: an on-line Google form and a paper form. We received 12 electronic responses and 2 paper responses, from which we extracted five data points for the investigation.

Two key issues arose from the data. First, the location and physical data associated with each response were not always adequate; for example, a trail rather than a precise location would be given because the hiker either did not have a Global Positioning System (GPS) or had a GPS but did not have a convenient way to record their position. Second, the responses did not always contain the direct comparison between forest and open areas that we were interested in; for example, one response described the snow conditions above tree line. We additionally 
observed that we lacked both spatial and temporal resolution in the year one dataset, but we attributed our sparse dataset to the "soft" roll-out in year one.

Feedback from the participants communicated a general sense of enthusiasm for the scope of the project, but also reflected some of the same data issues. Participants commented that they thought they understood what observations to collect in the field, but then were unsure when it came time to fill out the form upon their return. The most complete observations came from a participant who routinely makes multi-day treks in the mountains and specifically requested that we provide a paper form that could be used to make notes while in the field. Our assessment from our year one experience was that we needed to streamline the data collection and submission process, to provide more explicit instructions, and to recruit more participants.

Thus, we began year two with a change in focus from recording written observations to collecting geotagged photographs, improved educational materials, and an effort to recruit many more participants to generate more data in both time and space. The advantage of geotagged photographs is that the metadata embedded in the photograph includes the key information, including date and time, latitude and longitude, and elevation, and they are easy to take with a smartphone or GPS-enabled camera [Wilson et al., 2015] We updated our website and written materials with education related to the project and re-tooled directions to describe 1) how to take a geotagged photograph, 2) what to take the photos of, and 3) how to submit the photos. We created a 2-minute YouTube video describing the project and how to participate (https:/ /www.youtube.com/watch?v= JEmIV9vOXZ4). With the infrastructure in place, we reached out to the community via calls and emails to hiking organizations, emails to university and research groups, blog posts, presentations at conferences and professional meetings, and the personal networks of the research team. We embedded a Google Earth map into the project website to display observations as they were submitted next to our model predictions for the region. Judging from the abundant enthusiasm that we encountered, we prepared ourselves for numerous submissions and declared the public outreach goal of the project to be a success.

However, from the standpoint of usable data, the 28 photo submissions from 9 individuals that we received were less than we had hoped for, and the usability of the observations documented in photographs was highly variable. Participants in year two included hikers, mountaineers, and scientists doing other field research, and they provided positive feedback that they enjoyed contributing to the investigation. However, the range in the field of view of the photographs created challenges for data analysis, with submissions that ranged from landscape shots to photographs documenting local snowmelt features such as tree wells. With landscape shots, the geolocation of the photograph (i.e., the coordinates of the camera position) does not match the geolocation of the observation, nor indicate the topographic position of the subject (i.e., a north-facing versus south-facing slope). With close-up shots, the spatial context of the observations is not apparent, so drawing a conclusion about snow presence within a forest becomes difficult when looking at only a few trees. 
At the end of year two we substantially reconsidered our strategy and ultimately redesigned the project. To date, the observations that we had received were too sparse in both time and space to provide a meaningful way to bracket the difference in the timing of snow disappearance between the forest and the open. At best, photographs from a single location could tell us whether snow persisted longer in the forest or in the open but could not indicate the absolute difference in timing, which is critical to our investigation. We realized that even without issues of data quality, we would need many, many more observations for the approach of collecting geotagged photographs to be successful.

Upon reflection, we discovered a mismatch between the level of engagement and the numbers of participants that we needed for the dataset that we originally envisioned. We realized that our vision of participation fell between two common levels of citizen science engagement [Haklay, 2013]. We wanted participants to function at level 2 and make an informed decision about where to observe a fair comparison between snowpack in the forest and open and about the spatial scale at which to document that comparison. At the same time, we fundamentally needed a lot of data to bracket differential snowmelt timing over such a large region, which calls for a level 1 approach. Since the design of our project fell in between the two levels, the program did not successfully engage participants at either level. On one hand, our training resources and participation guidelines were not fully utilized by those who did participate which resulted in unusable data. Simultaneously, the extensive directions probably acted as a barrier to achieve widespread participation by making the process too complicated.

Additionally, a level 1 approach is perhaps most feasible when the subject of data collection is inherently interesting or photogenic, as in the case of birds or wildflowers. Snowy landscapes are frequently photographed, but close range views of melting snow have less general appeal. We briefly considered mining public repositories of photographs for the observations and locations of interest as previously tested for monitoring wildflower phenology on Mount Rainier [Wilson et al., 2015] However, we ruled this option out because the spatial scale of landscape photographs would limit our data quality, and the approach lacks a meaningful public outreach component We were left with a choice to make: focus on expanding participation in a simplified public project or focus in on training volunteers who would commit to making repeat observations in specific locations. Around the time that we were considering how to move forward, we became aware of the University of Idaho College of Natural Resources' McCall Outdoor Science School (MOSS), and quickly realized that a direct collaboration with an education program located in a region where we needed data could be a solution to meet both our data needs and our goal to involve public stakeholders. Thus, we shifted our focus from a public outreach to a collaboration, in which we would trade spatial coverage for informed participation and high temporal resolution via repeat observations.

\section{Year three: collaboration with MOSS}

From our first conversation it became apparent that a partnership between the research institutions and the MOSS would be mutually beneficial. MOSS is a 
residential outdoor science school serving more than 2500 students each year in week-long inquiry-based, place-based experiential science programs. Each week throughout the school year, groups of approximately $60-805^{\text {th }}-8^{\text {th }}$ grade students from a variety of locations throughout Idaho, Oregon and Washington travel to MOSS to spend a week attending school in the outdoors. Their instructors are graduate students enrolled in a graduate residency in environmental education through the University of Idaho's College of Natural Resources. The winter curriculum at MOSS focuses on snowpack dynamics, hydrology, winter ecology and energy balances, so the citizen science project was a natural complement to the existing curriculum. MOSS instructors lead field groups of 8-12 students into snow-covered, forested field sites on a weekly basis to collect snow data, resulting in groups who are well-positioned to constrain the timing of snow disappearance to a weekly temporal resolution.

The structured, educational data collection program implemented at MOSS is atypical from the standpoint of a traditional citizen science project in which volunteer citizens participate on an ad hoc basis. However, the program also very clearly fits the model for best practices related to involving citizens in scientific research that was developed by the Cornell Lab of Ornithology, which has famously led large-scale successful citizen science projects such as Project Feederwatch and the Great Backyard Bird Count [Bonney et al., 2009]. The Cornell citizen science framework served as a guide for developing the collaboration and provides guidance for reflection on successes and possible improvements. For our experience the first steps in the framework were already complete by the time we initiated the collaboration, including choosing a scientific question and forming the collaborative team.

The key step that was essential to complete together was to adjust and test protocols and materials for implementation in the MOSS program. This step was initiated via conversations about the nature of the data collection needs and the MOSS program, with both the research team and the MOSS team trying to understand how one would most easily and beneficially fit into the other. Since the MOSS program already includes instructor-led field excursions and field data recording on tablet computers, we decided to 1) standardize the locations which each instructor would visit each week so as to collect repeat observations in the same place, 2) design protocols to include taking photographs to record observations (Figure 1b), and 3) build a web-based form for data entry of estimated snow cover (Figure 1c). The research team provided initial protocols and drafts of web forms, with iterative feedback from the MOSS team as to the fit for their curriculum and logistics. The MOSS team was responsible for site selection and training of graduate students, with feedback and contributions from the research team via a video seminar.

By incorporating two forms of data collection in the protocols, including photographs and field-based estimates, we met the dual goals of collecting quality data and providing students a means by which to see their contribution to the project. The fraction of snow-covered area in each cardinal direction was estimated for the forested and open portions of each site, entered into the web form while in the field, and then automatically averaged and displayed as a time series plot (Figure 1c). As a subjective estimate, these numbers could then be validated via quantitative image analysis. 
Although the program seemed to be a perfect fit in many ways, the implementation into the MOSS curriculum was not without challenges. Each graduate instructor was responsible for visiting their site every other week, ideally with their students, but this did not always happen. Reaching a fixed site via snowshoes during field instruction was occasionally limited by students' physical abilities and stamina, as well as bad weather. Future integration of the data collection into instructor training will help to define it as an integral part of the educational experience rather than an additional task. Other challenges were encountered during the key step of recruiting participants for a citizen science program; in this case recruitment was easy since the data collection was integrated into the overall educational program. However, we suspect that the fact that participation was not entirely voluntary did occasionally result in a lack of motivation on the part of the instructors. Based on informal conversations with instructors we determined that their level of commitment varied between those who were motivated by the educational opportunity of collecting data relevant to a research investigation, to those with lower interest due to difficulty getting to the site, lack of understanding of the importance of the work, or lack of understanding how to incorporate the data collection into the rest of the field curriculum. For the future we would implement facetoface meetings between the research team and the graduate instructors to instill a sense of why the research matters. Although such a meeting is not possible with the student groups we are also considering making an introductory movie or meeting with students each week via video-conferencing technology.

From the perspective of MOSS, the citizen science program provides a promising way in which to connect educational activities to scientific practice. In addition to content exploration, the MOSS curriculum focuses on engaging students in the process of doing science, so partnering with research projects provides a way for students to connect with real scientists, and to see their data contribute to an on-going investigation. Previous studies have demonstrated that citizen science provides a platform on which science process skills can be practiced [Trumbull et al., 2000], and that participants show an increased support for science, scientific literacy, and sense of connection to the environment [Conrad and Hilchey, 2011]. Future iterations of this project at MOSS could include a formal evaluation of the participation experience of both students and graduate instructors in order to measure any or all of these learning outcomes and enhance our understanding of how to improve the program.

From a research perspective, we drastically limited the spatial scope of our citizen science project by abandoning our dispersed approach and partnering with MOSS for improved temporal data resolution. However, there are three key scientific reasons that this partnership proved more successful than previous years: 1) the data collected by students and instructors conformed to standardized protocols and were therefore high quality, 2) repeat observations through time were collected at a scientifically relevant time step, and 3) observations at the MOSS field site fill a spatial data gap for the project. For all of these reasons the MOSS dataset is being incorporated into the regional investigation, and represents important field data from a geographic and climatic data gap. As the only data from central Idaho, these observations record forest-snow interactions that are specific to a colder, continental/maritime climate regime. 
Overall, two key lessons arose from our three year experience with citizen science: 1) the data collection needed for the investigation needs to align with the strengths of the citizen science approach, and 2) the level of participation needed from the audience needs to align with both the regular activities and interests of the audience and the type of data being collected. For us, the first lesson was built into our approach when we recognized that this would be an ideal citizen science project. We are investigating processes that are difficult to model but straightforward to observe for someone who is in the right place at the right time. Thus, from the beginning we recognized that the data needed for this investigation would align well with a citizen science approach.

The second lesson was learned via the successes and failures over three years. In scaling up from year one to year two we focused on expanding participation in order to develop a spatially and temporally dense dataset. However, the flaw in this approach was that the level of engagement was mismatched with the numbers needed to achieve the desired dataset. Whereas we needed participants to collect data in an informed and thoughtful way, we also needed a large quantity of data that would be more readily achievable by engaging a large number of participants. Thus, we chose to compromise broad spatial coverage for quality data and changed our focus from engaging the public to engaging an organization that makes repeat trips to the same locations. We gave up the desired spatial resolution but achieved the temporal resolution and structured data collection that resulted in a usable dataset.

Through trial and error, we better defined the parameters for the collection of usable data, and we recognized that we either needed orders of magnitude more data in order to filter out the unusable data, or we needed our participants to follow specific instructions. We therefore honed in on the style of citizen science that resulted in success for the partnership and success for the investigation. In connecting with MOSS, we partnered with an organization that was already collecting similar data and that was seeking ways to make educational data collection more meaningful for students via connection with a research effort. Furthermore, the pilot program with MOSS has the potential to be replicated with other schools or organizations that are already making repeat visits to locations of interest throughout the snow season. Possible future partners include outdoor education centers located in mountainous or snowy regions, as well as organized recreational groups, such as snowmobiling clubs that make repeat trips along certain trails. In addition to the potential application of the approach described herein to other distributed snow studies, any scientific project to employ a citizen science for collecting distributed data may benefit from consideration of a spatially targeted approach.

In conclusion, our three year journey with citizen science resulted in meaningful connections with a wide range of interested individuals and organizations. In addition to the benefits of communicating current research to the public and supporting the educational goals of MOSS, the effort resulted in a temporally consistent dataset that filled a single spatial data gap in our regional analysis of forest influences on the timing of snow disappearance. 
Acknowledgments Primary support for the citizen science projects was provided by the National Science Foundation, CBET-0931780 and the Department of the Interior Northwest Climate Science Center (NW CSC) through Cooperative Agreement GS297A from the United States Geological Survey (USGS). The contents of this manuscript are solely the responsibility of the authors and do not necessarily represent the views of the NW CSC or the USGS. We are grateful to the many organizations and individuals that supported this effort each year, especially the instructors and students at the McCall Outdoor Science School, the Mountaineers, Anne Nolin, JoAnna Wendel, and Dave Tucker.

\section{References}

Bingaman, D. and Eitel, K. B. (2010). 'Boulder Creek Study: fifth graders tackle a local environmental problem through an inquiry-based project'. Science and Children. URL: https: //www. highbeam.com/doc/1G1-218880907.html.

Bonney, R., Cooper, C. B., Dickinson, J., Kelling, S., Phillips, T., Rosenberg, K. V. and Shirk, J. (2009). ‘Citizen Science: a Developing Tool for Expanding Science Knowledge and Scientific Literacy'. BioScience 59 (11), pp. 977-984. DOI: 10.1525/bio.2009.59.11.9.

Bonney, R., Shirk, J. L., Phillips, T. B., Wiggins, A., Ballard, H. L., Miller-Rushing, A. J. and Parrish, J. K. (2014). 'Citizen science. Next steps for citizen science'. Science (New York, N.Y.) 343 (6178), pp. 1436-1437. DOI: 10.1126/science. 1251554.

Borden, R. J., Cline, K. S., Hussey, T., Longsworth, G. and Mancinelli, I. (2007). 'A river runs through it: a college-community collaboration for watershed-based regional planning and education'. Human Ecology Review 14, pp. 90-100.

Buytaert, W., Zulkafli, Z., Grainger, S., Acosta, L., Alemie, T. C., Bastiaensen, J., De Bièvre, B., Bhusal, J., Clark, J., Dewulf, A., Foggin, M., Hannah, D. M., Hergarten, C., Isaeva, A., Karpouzoglou, T., Pandeya, B., Paudel, D., Sharma, K., Steenhuis, T., Tilahun, S., Van Hecken, G. and Zhumanova, M. (2014). ‘Citizen science in hydrology and water resources: opportunities for knowledge generation, ecosystem service management, and sustainable development'. Frontiers in Earth Science 2, pp. 1-21. DOI: 10.3389/feart.2014.00026.

Clark, M. P., Nijssen, B., Lundquist, J. D., Kavetski, D., Rupp, D. E., Woods, R. A., Freer, J. E., Gutmann, E. D., Wood, A. W., Gochis, D. J., Rasmussen, R. M., Tarboton, D. G., Mahat, V., Flerchinger, G. N. and Marks, D. G. (2015). 'A unified approach for process-based hydrologic modeling: 2. Model implementation and case studies'. Water Resources Research 51 (4), pp. 2515-2542. DOI: 10.1002/2015WR017200.

CoCoRaHS (2015). CoCoRaHS. Community Collaborative Rain, Hail and Snow Network. URL: http://www . cocorahs.org/.

Conrad, C. C. and Hilchey, K. G. (2011). 'A review of citizen science and community-based environmental monitoring: issues and opportunities'. Environmental Monitoring and Assessment 176 (1), pp. 273-291. DOI: 10.1007/s10661-010-1582-5.

Cooper, C. B., Dickinson, J., Phillips, T. and Bonney, R. (2007). 'Citizen science as a tool for conservation in residential ecosystems'. Ecology and Society 12 (2), p. 11. URL: http://www . ecologyandsociety. org/vol12/iss2/art11/.

Dickerson-Lange, S. E., Lutz, J. A., Martin, K. A., Raleigh, M. S., Gersonde, R. and Lundquist, J. D. (2015). 'Evaluating observational methods to quantify snow duration under diverse forest canopies'. Water Resources Research 51 (2), pp. 1203-1224. DOI: 10.1002/2014WR015744. 
Eick, C., Deutsch, B., Fuller, J. and Scott, F. (2008). 'Making Science Relevant: Water-Monitoring Programs Help Students Study Science While Protecting Local Waterways'. The Science Teacher 75 (4), pp. 26-29. URL:

https://www . questia.com/library/journal/1G1-182530794/making-science -relevant-water-monitoring-programs.

Elder, K. and Cline, D. (2003). CLPX-Ground: ISA Snow Depth Transects and Related Measurements. Boulder, Colorado U.S.A.: NASA National Snow and Ice Data Center Distributed Active Archive Center. DOI: 10.5060/D4MW2F23.

Ellis, C. R., Pomeroy, J. W. and Link, T. E. (2013). 'Modeling increases in snowmelt yield and desynchronization resulting from forest gap-thinning treatments in a northern mountain headwater basin'. Water Resources Research 49 (2), pp. 936-949. DOI: 10.1002/wrcr. 20089.

Essery, R., Rutter, N., Pomeroy, J., Baxter, R., Stähli, M., Gustafsson, D., Barr, A., Bartlett, P. and Elder, K. (2009). 'SNOWMIP2: An Evaluation of Forest Snow Process Simulations'. Bulletin of the American Meteorological Society 90 (8), pp. 1120-1135. DOI: 10.1175/2009BAMS2629.1.

Ford, K. R., Ettinger, A. K., Lundquist, J. D., Raleigh, M. S. and Hille Ris Lambers, J. (2013). 'Spatial heterogeneity in ecologically important climate variables at coarse and fine scales in a high-snow mountain landscape'. PLoS One 8 (6), e65008. DOI: 10.1371/journal.pone.0065008.

Galloway, A. W. E., Tudor, M. T. and Haegen, W. M. V. (2006). 'The Reliability of Citizen Science: A Case Study of Oregon White Oak Stand Surveys'. Wildlife Society Bulletin 34 (5). Ed. by West, pp. 1425-1429. DOI: 10.2193/0091-7648(2006)34[1425:TROCSA] 2.0.CO;2.

Haklay, M. (2013). 'Citizen Science and Volunteered Geographic Information: Overview and Typology of Participation'. In: Crowdsourcing Geographic Knowledge. Ed. by D. Sui, S. Elwood and M. Goodchild. Dordrecht, Netherlands: Springer, pp. 105-122. DOI: 10.1007/978-94-007-4587-2_7.

Howe, J. (2006). The Rise of Crowdsourcing. WIRED. URL: http://www. wired.com/2006/06/crowds/.

Kittredge, J. (1953). 'Influences of Forests on Snow in the Ponderosa, Sugar Pine, Fir Zone of the Central Sierra Nevada'. Hilgardia 22 (1), pp. 1-96. DOI: 10.3733/hilg.v22n01p001.

Lawless, J. G. and Rock, B. N. (1998). 'Student Scientist Partnerships and Data Quality'. Journal of Science Education and Technology 7 (1), pp. 5-13. DOI: 10.1023/A: 1022575914118.

Lowry, C. S. and Fienen, M. N. (2013). 'CrowdHydrology: crowdsourcing hydrologic data and engaging citizen scientists'. Ground Water 51 (1), pp. 151-156. DOI: 10.1111/j.1745-6584.2012.00956.x.

Lundquist, J. D., Dettinger, M. D. and Cayan, D. R. (2005). 'Snow-fed streamflow timing at different basin scales: Case study of the Tuolumne River above Hetch Hetchy, Yosemite, California'. Water Resources Research 41 (7), W07005. DOI: 10.1029/2004WR003933.

Lundquist, J. D., Dickerson-Lange, S. E., Lutz, J. A. and Cristea, N. C. (2013). 'Lower forest density enhances snow retention in regions with warmer winters: a global framework developed from plot-scale observations and modeling'. Water Resources Research 49 (10), pp. 6356-6370. DOI: 10.1002/wrcr. 20504.

Peckenham, J. M. and Peckenham, S. K. (2014). 'Assessment of Quality for Middle Level and High School Student-Generated Water Quality Data'. JAWRA Journal of the American Water Resources Association 50 (6), pp. 1477-1487. DOI: 10.1111/jawr.12213. 
Raleigh, M. S., Rittger, K., Moore, C. E., Henn, B., Lutz, J. A. and Lundquist, J. D. (2013). 'Ground-based testing of MODIS fractional snow cover in subalpine meadows and forests of the Sierra Nevada'. Remote Sensing of Environment 128, pp. 44-57. DOI: 10.1016/j.rse. 2012.09.016.

Riesch, H., Potter, C. and Davies, L. (2013). 'Combining citizen science and public engagement: the Open AirLaboratories Programme'. JCOM 12 (03), A03. URL: http://jcom.sissa.it/archive/12/3-4/JC0M1203\%282013\%29A03.

Rock, B. N. and Lauten, G. N. (1996). 'K-12th grade students as active contributors to research investigations'. Journal of Science Education and Technology 5 (4), pp. 255-266. DOI: 10.1007/BF01677123.

Rosenberg, K. V., Hames, R. S., Rohrbaugh Jr., R. W., Swarthout, S. B., Lowe, J. D. and Dhondt, A. A. (2003). Improving Habitat for Forest Thrushes. Cornell Lab of Ornithology. URL: http://static . birds . cornell. edu/conservation/thrush/.

Schon, J. A., Eitel, K. B., Bingaman, D. and Miller, B. G. (2014). 'Big Project, Small Leaders'. Science and Children 51 (9), pp. 48-54. DOI: 10.2505/4/sc14_051_09_48.

Silvertown, J. (2009). 'A new dawn for citizen science'. Trends in Ecology E Evolution 24 (9), pp. 467-471. DOI: 10.1016/j.tree.2009.03.017.

Trumbull, D. J., Bonney, R., Bascom, D. and Cabral, A. (2000). 'Thinking scientifically during participation in a citizen-science project'. Science Education 84 (2), pp. 265-275. DOI: 10.1002/(SICI) 1098-237X (200003) 84:2<265: :AID-SCE7>3.0.CO;2-5.

Wilson, A., Bacher, K., I., B., Lundquist, J., Rochefort, R., Theobald, E., L., W. and HilleRisLambers, J. (2015). 'Monitoring Wildflower Phenology using Traditional Science, Citizen Science, and Crowd Sourcing'. In Review. Park Science.

Susan E. Dickerson-Lange, is completing a doctoral dissertation at the University of Washington focused on the combined impacts of forest disturbance and climate change on snow and streamflow. With a professional background that includes research, teaching, community programs, and environmental consulting, she is particularly interesting in engaging land managers and public stakeholders in watershed science.

Civil and Environmental Engineering, University of Washington, Seattle, Washington, U.S.A. E-mail: dickers@uw.edu.

Karla Bradley Eitel, is Associate Professor in Natural Resources and Society at the University of Idaho (UI) and currently serves as Director of Education for the UI College of Natural Resources' McCall Outdoor Science School where she guides development of K12 curriculum and the graduate residency in place-based science, education and communication. Her research interests include incorporation of cutting edge science into K12 curriculum, STEM identity development in a place-based context and appropriate use of technology in outdoor learning environments.

McCall Outdoor Science School, College of Natural Resources, University of Idaho, McCall, Idaho, U.S.A. E-mail: keitel@uidaho.edu. 
Leslie Dorsey, is the Program Coordinator at the University of Idaho College of Natural Resources' McCall Outdoor Science School where she supervises graduate students in their role as field instructors for K12 students. Her previous position was Citizen Science Coordinator for Idaho Fish and Game and she brought her passion for citizen science to MOSS.

McCall Outdoor Science School, College of Natural Resources, University of Idaho, McCall, Idaho, U.S.A. E-mail: ldorsey@uidaho.edu.

Timothy E. Link, is a Professor of Hydrology in the College of Natural Resources at the University of Idaho (UI). He is also the director the UI Integrative Graduate Education Research Traineeship (IGERT) program in water resources, which is focused on training PhD students to address complex interactions and feedbacks in physical, ecological, and social systems resulting from the combined impacts of climate change and human population dynamics.

Department of Forest, Rangeland, and Fire Sciences, University of Idaho, Moscow, Idaho, U.S.A. E-mail: tlink@uidaho.edu.

Jessica D. Lundquist, is an Associate Professor in Civil and Environmental Engineering at the University of Washington. Her research interests focus on water in complex terrain, involving patterns in weather, climate, snow, vegetation, and runoff, particularly in the mountains of the western United States.

Civil and Environmental Engineering, University of Washington, Seattle, Washington, U.S.A. E-mail: jdlund@uw.edu.

Dickerson-Lange, S. E., Bradley Eitel, K., Dorsey, L., Link, T. E. and Lundquist, J. D. (2016). 'Challenges and successes in engaging citizen scientists to observe snow cover: from public engagement to an educational collaboration'. JCOM 15(01), A01. 